\title{
Examining speed versus selection in connectivity models using elk migration as an example
}

\author{
Angela Brennan (iD - Ephraim M. Hanks • Jerod A. Merkle • Eric K. Cole • \\ Sarah R. Dewey • Alyson B. Courtemanch $\cdot$ Paul C. Cross
}

Received: 28 November 2017/ Accepted: 30 March 2018/Published online: 26 April 2018

(C) The Author(s) 2018

\begin{abstract}
Context Landscape resistance is vital to connectivity modeling and frequently derived from resource selection functions (RSFs). RSFs estimate relative probability of use and tend to focus on understanding habitat preferences during slow, routine animal movements (e.g., foraging). Dispersal and migration, however, can produce rarer, faster movements, in which case models of movement speed rather than resource
\end{abstract}

Electronic supplementary material The online version of this article (https://doi.org/10.1007/s10980-018-0642-z) contains supplementary material, which is available to authorized users.

\footnotetext{
A. Brennan $(\bowtie) \cdot$ J. A. Merkle

Wyoming Cooperative Fish and Wildlife Research Unit, Department of Zoology and Physiology, University of Wyoming, Laramie, WY, USA

e-mail: angie_brennan@hotmail.com

E. M. Hanks

Department of Statistics, Pennsylvania State University, State College, PA, USA

E. K. Cole

U.S. Fish and Wildlife Service, National Elk Refuge, Jackson, WY, USA
}

S. R. Dewey

National Park Service, Grand Teton National Park,

Moose, WY, USA selection may be more realistic for identifying habitats that facilitate connectivity.

Objective To compare two connectivity modeling approaches applied to resistance estimated from models of movement rate and resource selection.

Methods Using movement data from migrating elk, we evaluated continuous time Markov chain (CTMC) and movement-based RSF models (i.e., step selection functions [SSFs]). We applied circuit theory and shortest random path (SRP) algorithms to CTMC, SSF and null (i.e., flat) resistance surfaces to predict corridors between elk seasonal ranges. We evaluated prediction accuracy by comparing model predictions to empirical elk movements.

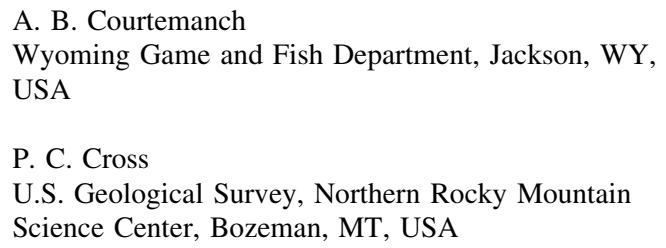

A. B. Courtemanch

Wyoming Game and Fish Department, Jackson, WY, USA

P. C. Cross

U.S. Geological Survey, Northern Rocky Mountain Science Center, Bozeman, MT, USA 
Results All connectivity models predicted elk movements well, but models applied to CTMC resistance were more accurate than models applied to SSF and null resistance. Circuit theory models were more accurate on average than SRP models.

Conclusions CTMC can be more realistic than SSFs for estimating resistance for fast movements, though SSFs may demonstrate some predictive ability when animals also move slowly through corridors (e.g., stopover use during migration). High null model accuracy suggests seasonal range data may also be critical for predicting direct migration routes. For animals that migrate or disperse across large landscapes, we recommend incorporating CTMC into the connectivity modeling toolkit.

Keywords Migration - Movement models - Wildlife corridors · Landscape connectivity $\cdot$ Resistance modeling · Corridor conservation · Greater Yellowstone area $\cdot$ Landscape conductance

\section{Introduction}

Connectivity modeling and corridor conservation are aimed at predicting and preserving space for animal movement, gene flow, and ecological processes to occur across landscapes affected by habitat loss and fragmentation (Chetkiewicz et al. 2006; Hilty et al. 2006). Corridors are also expected to support range shifts for species responding to climate change (Hilty et al. 2006; Heller and Zavaleta 2009). Thus, identifying corridors and improving connectivity have become important conservation tools to facilitate climate change resiliency, improve population viability, conserve biodiversity and uphold the ecological value of protected areas (Bennett 2003; Hilty et al. 2006; Heller and Zavaleta 2009).

Recent studies argue, however, that corridors could fail to protect rare, fast and directed movements that can be important to ecological processes (e.g., dispersal and migration), because popular connectivity modeling methods rely on weak relationships to these types of movement behaviors (e.g., Elliot et al. 2014; Zeller et al. 2014; Abrahms et al. 2016; Keeley et al. 2017). Circuit theory and cost-distance models, for example, require gridded input describing landscape resistance to movement (i.e., the cost of moving through each cell in the landscape; Zeller et al. 2012), but typically resistance is derived from subjective relationships with habitat suitability (Beier et al. 2008; Chetkiewicz and Boyce 2009). Resource selection functions (RSFs) used to estimate habitat suitability also tend to be biased towards understanding habitat preferences during slow, routine movements where more animal relocations are recorded (e.g., foraging areas), instead of habitats associated with rarer, faster and more directed movements (as suggested in Van Dyck and Baguette 2005; Elliot et al. 2014; Zeller et al. 2014; Blazquez-Cabrera et al. 2016). Consequently, existing methods for estimating resistance could result in conservative corridor predictions that exclude areas important for connectivity.

A common framework for estimating resistance follows three main steps: (1) parameterize a RSF based on animal location data with gridded landscape covariates $x$, (2) generate an index of habitat suitability $\hat{w}$ from the RSF by combining the estimated parameters $\beta$ and covariates $x: \hat{w}(x)=\exp \left(\beta_{1} x_{1}+\beta_{2} x_{2}\right.$ $+\cdots+\beta_{j} x_{j}$ ), and (3) calculate resistance $r$ as a negative linear or nonlinear function of habitat suitability via $r=1-\hat{w}(x)$ or $r=1 / \hat{w}(x)$, respectively (e.g., Chetkiewicz and Boyce 2009, Zeller et al. 2012, Squires et al. 2013, McClure et al. 2016). RSFs are generally employed to compare habitat characteristics at 'used' and 'available' animal locations to quantify resource selection, map relative probability of use and predict species distributions (Manly et al. 2002). In the framework described above, however, RSFs are used to generate a gridded landscape resistance surface following a linear or nonlinear transformation of habitat suitability. Either transformation in this step will result in similar low and high resistance values, but vastly different values of intermediate resistance (Trainor et al. 2013; Keeley et al. 2016, 2017). And while the transformation used can be informed by targeted movement type (Trainor et al. 2013; Keeley et al. 2016, 2017), ultimately which is used remains subjective (Chetkiewicz et al. 2006; Beier et al. 2008). Recent studies have evaluated the effect of linear and multiple nonlinear transformations of habitat suitability on connectivity model performance, but typically the relationship to resistance is not examined nor formally justified (Trainor et al. 2013; Keeley et al. 2016, 2017). 
Also when using RSFs, areas where animals make rare, fast and directed movements (e.g., long distances between relocations) may have fewer 'used' locations and thus appear to have lower habitat suitability and higher resistance compared to areas where routine, slow or tortuous movements occur (e.g., short distances between relocations) (as in Johnson et al. 2002). In other words, areas with fewer locations can appear to have lower overall habitat suitability using a RSF, even though they facilitate fast movement and function as a conduit for migration or dispersal (because faster movements can be an important component of these processes; Johnson et al. 2002 and Van Dyck and Baguette 2005). Thus, landscape resistance estimated from a RSF could bias connectivity models toward routine movements, while an analysis of movement speed could yield the opposite conclusions about habitat effects on movement. Parameterizing a RSF for different movement behaviors (e.g., dispersal versus foraging) can reduce bias toward one type of movement, but it requires additional steps or behavioral observations to separate the data by a specific behavior (Chetkiewicz et al. 2006; Elliot et al. 2014; Zeller et al. 2014; Abrahms et al. 2016; Blazquez-Cabrera et al. 2016). Explicitly modeling movement rate on the other hand, may be an alternative to RSFs for estimating resistance to rare, fast and directed movements and help identify habitats that facilitate connectivity.

The continuous time Markov chain model (CTMC) was recently developed to model movement rate across a gridded surface and examine location-based and directional drivers of animal movement along a pathway (Hanks et al. 2015). A CTMC model uses fine scale animal location data converted into a continuous-time discrete space path on a gridded surface. This conversion compresses the data to a scale relevant to the resolution of gridded landscape covariates, where every grid cell along each individual's path is associated with a residence time $\tau$ determined by the number and fix rate of animal locations within each cell. Using a latent variable representation of the CTMC path (i.e., cells on path $=1$ and cells neighboring the path $=0$ ) and $\tau$ as the exposure term, inference can be made about landscape covariate effects on movement rate $1 / \tau$ within a Poisson generalized linear model framework (Hanks et al. 2015). Model parameters and gridded landscape covariates can then be combined to estimate the rate of transition between neighboring grid cells, where larger transition rates correspond to faster movement rates. Grid cell transition rates estimated using this approach relate directly to conductance or $1 /$ resistance in electrical circuit theory (Hanks and Hooten 2013).

As CTMC models consider the effects of the path on movement rate, they capture a fundamentally different movement paradigm than RSFs and could improve our understanding of resistance for rare, fast and directed movements. In this study, we evaluated CTMC as an alternative to RSFs for estimating resistance and modeling connectivity, but rather than use traditional RSFs for this comparison, we used a movement-based RSF (i.e., step selection functions [SSFs]) to estimate resource selection along the movement pathway, conditional on local availability estimated from empirical step lengths and angles (Fortin et al. 2005). With an emphasis on resource selection during movement and a more realistic estimation of availability, SSFs are arguably more appropriate than traditional RSFs (Zeller et al. 2012) and are becoming preferred for estimating resistance in connectivity model applications (e.g., Keeley et al. 2016, 2017, Panzacchi et al. 2016, Zeller et al. 2016).

We examined CTMC and SSFs in connectivity models using GPS collar data from elk (Cervus canadensis) that migrate during the spring from the National Elk Refuge to Yellowstone National Park in western Wyoming. Here, spring migration typically extends $75-100 \mathrm{~km}$, crossing national forest, some private and agricultural land, roadways, strong elevational gradients, and variable forest cover, forage quality and water availability. Such migrations support other important ecological processes (e.g., predator-prey interactions, competition and energy transfer) (Houston 1982), but evidence suggests that elk migratory patterns are shifting and that the proportion of elk that migrate is shrinking (e.g., Middleton et al. 2013; Cole et al. 2015). Changing migrations is also a concern for other ungulate species across the globe (as summarized in Middleton et al. 2013), and thus understanding landscape attributes important to movement and identifying movement corridors are important steps toward preserving migration potential.

We parameterized a CTMC and SSF with landscape attributes, expecting to reach opposite conclusions regarding elk habitat preferences because elk may move faster through relatively unsuitable terrain. 
We used these models to generate different resistance surfaces and applied two connectivity model algorithms (circuit theory and shortest random path [SRP]) to predict spring elk migration corridors. We used circuit theory and SRP to model the flow of movement through a resistance surface from a source to a target node. Circuit theory is based on a random walk model and thus assumes that individuals have no knowledge of the landscape beyond their immediate surroundings. The SRP algorithm on the other hand uses a parameter $\theta$ to constrain random exploration so that movement across a gridded landscape is neither a random walk nor fully optimized as in cost-distance methods which assume perfect knowledge of the landscape (Panzacchi et al. 2016).

We evaluated corridor prediction accuracy by comparing circuit theory and SRP model values to elk locations from individuals held out of the models. We expected connectivity models applied to CTMC resistance (hereafter referred to as CTMC-informed models) to predict elk migration corridors more accurately than connectivity models applied to SSF resistance (hereafter referred to as SSF-informed models) because CTMC may better reflect habitat use during migratory movements than a SSF based on all movement types. Because elk movements during migration suggest some knowledge of the landscape (McClure et al. 2016), we also expected SRP models to have higher prediction accuracy than circuit theory models. Finally, we questioned whether corridors could be predicted using models informed only by the start and end of migration, and no information on resistance. Therefore, we evaluated the added value of landscape resistance in predicting elk migration corridors by comparing CTMC and SSF-informed connectivity models to null models (i.e., flat resistance surface, where all grid cells $=1$ ).

\section{Methods}

\section{Study dataset}

We obtained elk relocation data (i.e., 555,256 GPS locations from 119 GPS-collared adult female elk) from the U.S. Fish and Wildlife Service, Grand Teton National Park and Wyoming Game and Fish Department. We focused our analysis on 17 elk that migrated from the National Elk Refuge to Yellowstone National
Park (Fig. 1) during the spring as a way to focus our analysis on one type of migration (e.g., spring) and on long distance migrators with similar winter and summer ranges. The full focal elk dataset contained data collected during all months (104,913 locations; data can be downloaded from Brennan et al. 2018), but was subset to include data from only May and June for most analyses. This spring dataset included 11,254 recorded locations and 25 elk-years from 2006 through 2015 (17 individuals monitored for 1-2 years), with variable fix rates but with most relocations occurring at two, four and $8 \mathrm{~h}$ intervals (see Online Resources 1 for details). We split the spring dataset into a model training set (all spring locations for 13 randomly selected elk) to build models and a model validation set (all spring locations for the four remaining elk) to examine connectivity model prediction accuracy.

\section{Landscape covariates}

We parameterized a continuous time Markov chain model (CTMC) and step selection function (SSF) with ten gridded landscape covariates hypothesized to affect elk movement in western Wyoming including three anthropogenic variables (agricultural land, private land, and major roads) and seven natural variables (forage quality, elevation, forest cover, aspect, slope, terrain roughness, and distance to water) (e.g., Fortin et al. 2005; Merkle et al. 2017a). We created grids of agricultural land, forest cover and water from the National Land Cover Database. We rasterized private land polygons obtained from Surface Management Agency spatial data. We created a grid of highways from Topologically Integrated Geographic Encoding and Referencing (TIGER) data to examine the effect of major roads. For forage quality we calculated normalized difference vegetation index (NDVI) (Pettorelli et al. 2005) from 8-day surface reflectance data recorded by the Moderate Resolution Imaging Spectroradiometer (MODIS) at a 250-m resolution, and then extracted NDVI values nearest in date to the date of each GPS location. We used a digital elevation model for elevation and to derive slope, terrain roughness and aspect. Instead of using aspect directly, we converted the grid into a continuous representation of south-ness scaled from -1 (north) to 1 (south) using $-\cos (($ aspect $\times \pi) / 180)$. We resampled all covariate grids from their original resolution to $500 \mathrm{~m}$ for computational efficiency, and because $500 \mathrm{~m}$ was 
Fig. 1 Map of the study area and elk GPS locations for 17 elk that migrated between the National Elk Refuge and Yellowstone National Park (YNP). GTNP Grand Teton National Park

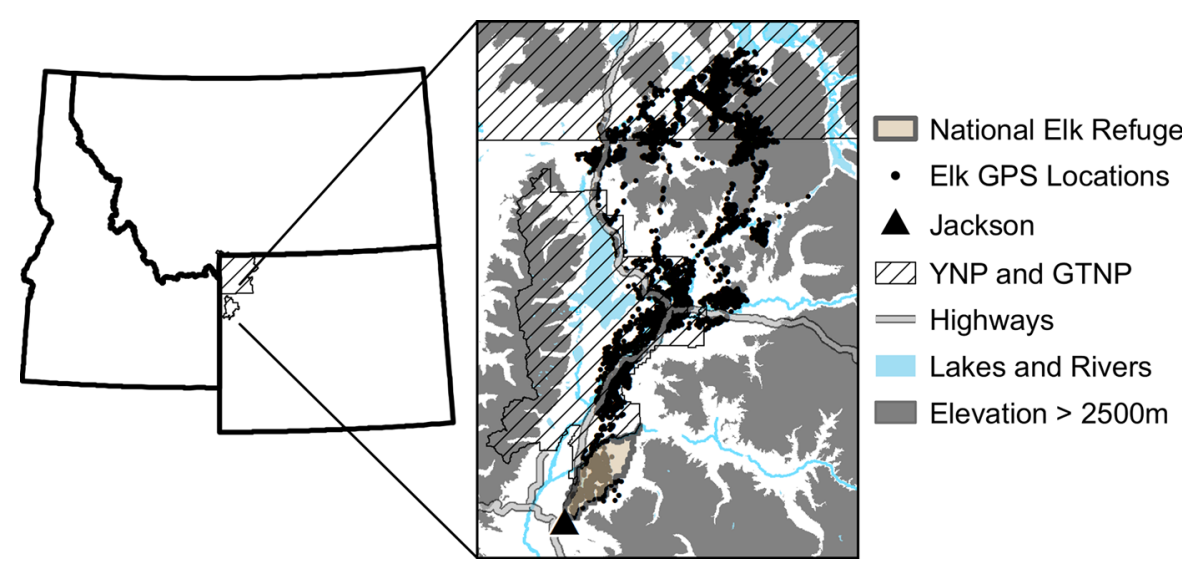

the median distance elk traveled over a relatively short time frame $(6-8 \mathrm{~h})$. We converted binary categorical variables to a continuous scale (e.g., new pixel value $=$ the average of focal pixel and four nearest pixel values). Grid processing methods and references for all covariates are described in Online Resources 2.

\section{CTMC}

We converted the full focal elk GPS dataset into a compressed CTMC path for each elk (using all months for the 17 focal migratory elk to avoid large jumps across years). Grid cells along these CTMC paths were associated with a residence time $\tau$ determined by the number and duration of GPS locations within each cell. When the interval and distance between GPS fixes result in skipped-over grid cells along the pathway, path imputation or linear interpolation can be employed to estimate the entire path between occupied grid cells (Hanks et al. 2015; Scharf et al. 2017). We used linear interpolation in this case because distances between GPS fixes were short relative to grid cell resolution (see Online Resources 1 for median distances). We created a latent variable from the CTMC path, where cells on the path were assigned a value of one and neighboring cells (using a rook's neighborhood) were assigned a value of zero. We extracted landscape covariate values for all of these cells and then subset the data to include locations from only the months of May and June and for only the 13 elk identified in the model training set. With the latent variable and residence time $\tau$ as the exposure term (used to convert count data into a rate via count/exposure), we were able to use a Poisson generalized linear model to evaluate covariate effects on spring movement rate $(1 / \tau)$. We used the 'СТмCMOVE' package (Hanks 2017) in the R environment for statistical computation ( $\mathrm{R}$ Core Development Team 2017) to create the CTMC path and latent variable.

Prior to estimating CTMC parameters, we examined correlations between covariates, and excluded slope and terrain roughness from the analysis because they were strongly correlated with elevation (Pearson's correlation coefficient $r>0.60$ ). The remaining eight covariates were included in the Poisson generalized linear model (Table 1).

We were also interested in examining the effect of elevation and forest cover on directional bias of elk movement, because elk in our study tend to migrate toward higher elevation in the spring, but may also move away from forested areas where deep snow can persist into late summer. To model directional bias using the 'CTMCMOVE' package in R (Hanks 2017), a transition vector $e_{i j}$ was created for each grid cell transition between cell $i$ and $j$ that points in the direction that the animal moved: $e_{i j}=\left(x_{j}-\mathrm{x}_{i}, \mathrm{y}_{j-}\right.$ $-\mathrm{y}_{i}$ ) (where $\mathrm{x}=$ longitude and $\mathrm{y}=$ latitude). A gradient vector was also created for a particular covariate of interest that points in the direction of the steepest increase in that covariate at cell $i$ (Hanks et al. $2011,2015)$. The product of the transition and gradient vectors indicates whether an animal is moving toward (product is positive) or away (product is negative) from the gradient, and can be added as a predictor in the generalized linear model (Hanks et al. 2011, 2015). Using this method, we examined the effect of directional bias from elevation and forest cover gradients on elk movement rate. We also accounted for 
Table 1 Estimated continuous time Markov chain (CTMC) and step selection function (SSF) coefficients and standard errors

Bold values correspond to
$p$ values $<0.05$
$S E$ standard errors
${ }^{\text {aa }}$ Scaled and centered
covariates
covariates

Bold values correspond to $p$ values $<0.05$

directional persistence (i.e., when the direction of movement is influenced by the direction of the previous move) in the CTMC model by including a correlated random walk term created from the product of the transition vector $e_{i j}$ described above and a vector that points in the direction of the previous move (Hanks et al. 2011, 2015). Positive values indicate that the animal's current move is in the same direction as the previous move.

With the addition of the directionality terms there were eleven total predictors in the CTMC model: eight landscape covariates, two covariates describing directional bias (referred to as 'elevation gradient' and 'forest cover gradient'), and one correlated random walk term (Table 1). We scaled and centered landscape and directional bias covariates (by subtracting the mean and dividing by the standard deviation) to compare the relative importance of the covariates to spring elk movement, and then fit the Poisson generalized linear model in R. We also estimated CTMC model parameters without scaling and centering the covariates to predict movement rate between adjacent cells of the gridded landscape (equivalent to conductance, or $1 /$ resistance, in electrical circuit theory; Hanks and Hooten 2013). We did not examine random effects for individual elk here because we were interested in estimating population-level resistance to movement using point estimates of model parameters, but CTMC models can be fit using Poisson regression with random effects.
To prepare the $1 /$ resistance surface from CTMC parameters and covariates, we first created an $n \times n$ matrix using the 'CTMCMOVE' package in $\mathrm{R}$ (Hanks 2017) where each row and column in the matrix represent a grid cell $n$ (e.g., column 1 and row 1 correspond to grid cell 1 ; column 1 and row $2 \ldots$ $n$ correspond to all potential neighbors of grid cell 1 ). We predicted movement rate $m$ between each grid cell and it's eight adjacent neighbors $k$ (i.e., king's neighborhood) by combining the unscaled parameters $\delta$ and gridded covariates $x$ using $m_{k}=\exp \left(\delta_{1} x_{1 k}+\delta_{2-}\right.$ $x_{2 k}+\cdots+\delta_{j} x_{j k}$ ). We did not include the correlated random walk parameter in this step because this parameter was used to account for autocorrelation in the relocation data and was not informed by landscape characteristics. For the NDVI grid used in this step, we used NDVI calculated from a MODIS 8-day, 250-m surface reflectance panel for 17 May 2014 that was resampled to a $500 \mathrm{~m}$ resolution. We chose this panel because mid-May NDVI represents forage quality during the time when most elk from the National Elk Refuge will be migrating. The resulting movement rate matrix (hereafter referred to as conductance matrix) was used in the shortest random path (SRP) connectivity model. For the circuit theory connectivity model, we converted the conductance matrix into a gridded conductance surface where the value of every grid cell was calculated as the mean conductance between that grid cell $n$ and all of its adjacent neighbors (i.e., mean of the non-zero entries in column 
$n$ of the conductance matrix), and then we rescaled the grid cell values to range between 0.001 and 1 (using 0.001 rather than 0 to avoid undefined resistances when resistance $=1 /$ conductance). Code to recreate CTMC paths, conductance matrices and gridded surfaces is provided in Online Resources 3.

For null model resistance, we created a grid with the same spatial extent and resolution as the CTMC conductance surface, but set all grid cell values equal to 1 to create a flat surface with no variation in resistance across the landscape. We included null models in our analysis to examine corridor predictions when only source and target nodes are known and understand the added value of landscape resistance on corridor predictions after comparison to models informed by resistance.

\section{Step selection functions}

Step selection functions (SSFs) are used to explicitly model correlation in successive GPS locations and study resource selection by animals moving across the landscape, conditional on local availability of resources (Fortin et al. 2005; Thurfjell et al. 2014). SSFs compare 'used' steps, representing pairs of consecutive observed locations, to random 'available' steps from the same starting point (Fortin et al. 2005). We rarefied the spring model training set of elk GPS data (rarefied $n=2859$ ) to obtain equal 8 -h intervals (i.e., step lengths), because the 8-h interval ensured that the median distance moved between locations $(533 \mathrm{~m})$ was roughly equivalent to the resolution of the covariate data (Thurfjell et al. 2014). For each used step we drew a random sample of 20 available steps from the empirical distribution of step lengths and turning angles. We identified ending coordinates of used and available steps and extracted covariate values for those locations. We used end points rather than averaging covariate values along each step to create a habitat suitability surface directly from multiplying selection coefficients and landscape covariates (rather than attempting to adjust surface values for how covariates change along steps).

Prior to model fitting, we examined correlations between all covariate values. As with the CTMC analysis described above, we excluded slope and terrain roughness because they were strongly correlated with elevation (Pearson's correlation coefficient $r>0.60$ ). With the remaining covariates (Table 1), we used conditional logistic regression to estimate selection parameters in a matched case-control design, where strata were assigned to each matched set of used and available steps (Thurfjell et al. 2014). We also included step length as a variable to reduce potential bias in parameter estimates associated with dependence between used and available steps (Forester et al. 2009). We conducted this analysis using the 'ClOGIT' function in the 'SURVIVAL' package in $\mathrm{R}$ (Therneau 2017).

We created an index of habitat suitability $\hat{w}$ by combining the estimated SSF parameters $\beta$ and landscape covariates $x$ using $\hat{w}(x)=\exp \left(\beta_{1} x_{1}+\beta_{2} x_{2}\right.$ $+\cdots+\beta_{j} x_{j}$ ) (e.g., Squires et al. 2013). This surface described population-level habitat suitability, as it is related to the long-term average selection of resources, assuming all terrain is available. We rescaled habitat suitability surface values to range from 0 to 0.999 and used a negative linear transformation to estimate resistance: $r=1-\hat{w}(x)$ (we used 0.999 as the upper habitat suitability value instead of 1 to avoid resistances of 0 when resistance $=1-$ habitat suitability).

\section{Connectivity models}

We evaluated elk connectivity between winter and summer range using circuit theory and shortest random path (SRP) algorithms. Circuit theory has been used to model connectivity as the flow of electrical current across a resistance surface (McRae et al. 2008) and is mathematically equivalent to the flow of animals moving in a CTMC random walk, with movement rate equal to $1 /$ resistance. Circuit theory predictions describe the probability of a random walker moving between grid cells as it travels from a source node to a ground node across a gridded resistance surface, and are generated under the assumption that travel route redundancy (i.e., multiple travel routes) between nodes can improve flow (i.e., animal movement) and boost connectivity (McRae et al. 2008).

We evaluated the SRP algorithm (Saerens et al. 2009; Kivimäki et al. 2014) for modeling elk migration because this method combines properties of optimized movement (e.g., cost-distance methods) and random walk theory (Panzacchi et al. 2016; van Etten 2017). The SRP approach estimates the probability of passage through each grid cell between source and target nodes as a function of a transition matrix 
and parameter $\theta$ used to control the balance of optimization and random exploration (Panzacchi et al. 2016; van Etten 2017). Here, transition matrices describe the ease of moving between adjacent grid cells and thus correspond to landscape conductance or permeability. The SRP method operates similar to optimized methods when $\theta$ values are large and similar to random walk methods when $\theta=0$ (for details see Panzacchi et al. 2016, van Etten 2017), but the overall effect of $\theta$ also depends on the number of grid cells and the average conductance across the grid (Panzacchi et al. 2016). In our case, we set all grids as equivalent in size and resolution and we adjusted the magnitude of $\theta$ based on average conductance for comparability among SRP models (e.g., when average conductance $=1, \theta$ was set to 0.001 and when average conductance $=10, \theta$ was set to 0.01$)$. We did not test multiple values of $\theta$ because our goal was to compare predictions from models with some constraint on random exploration to circuit theory models (but see Panzacchi et al. 2016 for an example of calibrating $\theta$ ).

Both circuit theory and SRP algorithms model connectivity between source and target nodes. In our study, the source node described the centroid of elk winter range on the National Elk Refuge and the target node described the centroid of elk summer range in Yellowstone National Park. We identified winter range using an $80 \%$ minimum convex polygon of all spring model training and validation elk locations in February, and summer range using an $80 \%$ minimum convex polygon of all elk locations in July.

We used Circuitscape (version 4.0; www. circuitscape.org) to apply the circuit theory algorithm to CTMC conductance, SSF resistance, and null resistance surfaces. We did not convert CTMC conductance into resistance because Circuitscape can be modified to make this conversion internally (McRae et al. 2013). We rescaled the resulting connectivity maps to range between 0 and 100, and then reclassified grid cell values into percentiles ranging from 1 to 99 . Percentiles describe the connectivity value below which a given percentage of the values occurred (e.g., $70 \%$ of the connectivity values occur below the 70th percentile). We used percentiles to identify the 'best' habitats for spring elk movement (i.e., upper percentiles) and to effectively compare predictions among the connectivity model scenarios (Morris et al. 2016) (but see Online Resources 4 for raw probabilities).
We used the 'PASSAGE' function in the 'GDISTANCE' package in R (van Etten 2017) to apply the SRP algorithm to the CTMC conductance matrix. We assigned $\theta$ to 0.001 to constrain random exploration around the shortest path. For the SSF comparison, we created a transition matrix (similar to CTMC conductance matrix) that describes the ease of transitioning from one grid cell to another, where each row and column in the $n \times n$ matrix represented a grid cell $n$ in the habitat suitability surface. Transition values in the matrix were calculated as the mean of habitat suitability for each pair of adjacent cells in a king's neighborhood (e.g., column 1 and row 2 represents the mean habitat suitability of grid cell 1 and adjacent grid cell 2). We used this transition matrix to index landscape permeability rather than conductance, as there is no formal mathematical relationship between habitat suitability and conductance. We applied the SRP algorithm with $\theta$ assigned to 0.01 and the seasonal range nodes previously described. Here, we used a $\theta$ value of 0.01 instead of 0.001 because the average landscape permeability value was an order of magnitude larger than average CTMC conductance. Code to replicate SRP models (with CTMC conductance as an example) is provided in Online Resources 3.

Following the method above, we created a null model transition matrix from the null model resistance surface, and then applied the SRP algorithm with $\theta$ assigned to 0.001 . Transition matrices were created using the 'TRANSITION' function in the 'GDISTANCE' package in R (van Etten 2017). We reclassified grid cell values from all resulting SRP connectivity models into percentiles ranging from 1 to 99 . Finally, we validated and compared all SRP and circuit theory model predictions by quantifying the cumulative percent of elk locations in each connectivity percentile for each elk in the spring validation set. Similar methods (and others) have been used elsewhere to evaluate connectivity model prediction accuracy (e.g., McClure et al. 2016).

\section{Results}

Negative CTMC coefficients for landscape covariates depict slower movement in grid cells with higher covariate values, and for gradient covariates, negative coefficients depict biased movement with preference 
for movement in the direction toward lower covariate values. Positive CTMC coefficients depict the opposite response in movement. CTMC coefficients suggest elk moved slower through grid cells with higher elevations and more agricultural land, major roads and southerly aspects; and that elk moved faster through grid cells with more forest cover and a further distance from water (Table 1). Of these landscape covariates, elevation had the largest effect on movement rate, while private land and forage quality had the weakest effects. The two coefficients describing directional bias suggest elk were more likely to move in the direction of decreasing elevation and forest cover, but the $95 \%$ confidence intervals for elevation gradient overlapped zero $(-0.088$ [ $-0.349,0.173]$; Table 1$)$. This lack of evidence for biased movement toward increasing elevation was unexpected, but an example CTMC path demonstrated that elk can move toward and away from steep elevations while also migrating to a higher elevation overall (Online Resources 5).

Positive SSF coefficients depict selection for a particular variable (i.e., more suitable habitat) and negative coefficients suggest avoidance (i.e., less suitable habitat). SSF coefficients suggest elk selected grid cells with higher elevations and forage quality, and more agricultural land, southerly aspects and major roads (Table 1). On the other hand, elk tended to avoid grid cells with high values of forest cover and private land, and grid cells far from water (but the effect size of distance to water was small and $95 \%$ confidence intervals overlapped zero; Table 1). Of these variables, forage quality, forest cover and elevation had the strongest effects on elk resource selection, while agricultural land, south-ness, major roads and distance to water had the weakest effects.

As expected, CTMC and SSF coefficients for most covariates were estimated to have opposite signs. This was not the case for coefficients for private land and NDVI, though these appear to be weak or poorly estimated in the CTMC model (Table 1). The similarity in sign for private land and NDVI, and potential similarity for distance to water from the SSF (having 95\% confidence intervals that overlapped zero), may suggest some overlap in areas that elk preferred for resource use and fast movement.

All connectivity models depicted high probabilities of movement immediately surrounding source and target nodes and relatively higher probability immediately between the nodes (Fig. 2). CTMC and SSF- informed connectivity models identified pinch points and depicted more detail overall than null model maps (Fig. 2). Overall, there was significant overlap in prediction accuracy across all connectivity model scenarios and the majority of validation elk locations (greater than 60\%) were found in the upper 30th percentile grid cells (i.e., percentiles 70-99) (Figs. 3, 4). CTMC-informed models, however, were $7-10 \%$ more accurate than SSF-informed and null models when looking at the median number of elk locations found in these upper percentiles (Fig. 4). Among CTMC-informed models, circuit theory demonstrated more consistent and higher overall accuracy than SRP at predicting elk movement (Figs. 3, 4). The SSFinformed and null models predicted elk locations with similar accuracy (Figs. 3, 4).

\section{Discussion}

We examined continuous time Markov chain models (CTMC) as an alternative to step selection functions (SSFs) for understanding drivers of elk movement during the spring migration, estimating landscape resistance and predicting migration corridors. We evaluated the CTMC approach because it predicts animal movement rate between neighboring grid cells and relates directly to conductance (or 1/resistance) in electrical circuit theory (Hanks et al. 2015). The relationship between SSFs and resistance, on the other hand, is subjective and not formally supported (Chetkiewicz et al. 2006; Beier et al. 2008). Our comparison of CTMC and SSF coefficients revealed opposite landscape effects on movement speed and selection for most covariates in our analysis, providing evidence that animals can move faster through terrain identified by SSFs as 'less-suitable' and slower through terrain identified as 'suitable'. CTMC-informed connectivity models were more accurate than SSF-informed models at predicting areas where elk in our study moved during the spring migration (Fig. 4). This result suggests that studies of movement speed could be used to understand habitats important for rare, fast and directed movements to help identify areas that facilitate connectivity.

Though CTMC-informed connectivity models performed best at predicting elk migration corridors in our study, SSF-informed models still predicted elk locations with a high level of accuracy and with more 
Fig. 2 a Continuous time Markov chain (CTMC) paths generated from 13 model training elk, b GPS locations for four validation elk, and $\mathbf{c}-\mathbf{h}$ connectivity maps predicting the probability of elk movement through each grid cell between seasonal range centroids. Connectivity model values were binned into percentiles from 1 through 99. Connectivity maps depict model predictions for $\mathbf{c}-\mathbf{e}$ circuit theory and $\mathbf{f}-\mathbf{h}$ shortest random path algorithms applied to CTMC, step selection function (SSF), and null (NULL) resistances. The southern seasonal range centroid (elk winter range) occurred on the National Elk Refuge and the northern seasonal range centroid (elk summer range) occurred in Yellowstone National Park (YNP). GTNP Grand Teton National Park
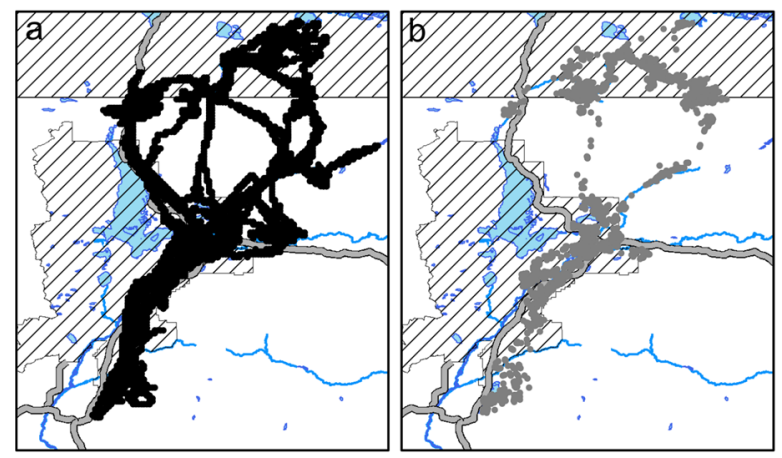

CTMC Paths:

- Model Training Elk

GPS Points:

Validation Elk

E Y YNP and GTNP

Lakes and Rivers

= Highways
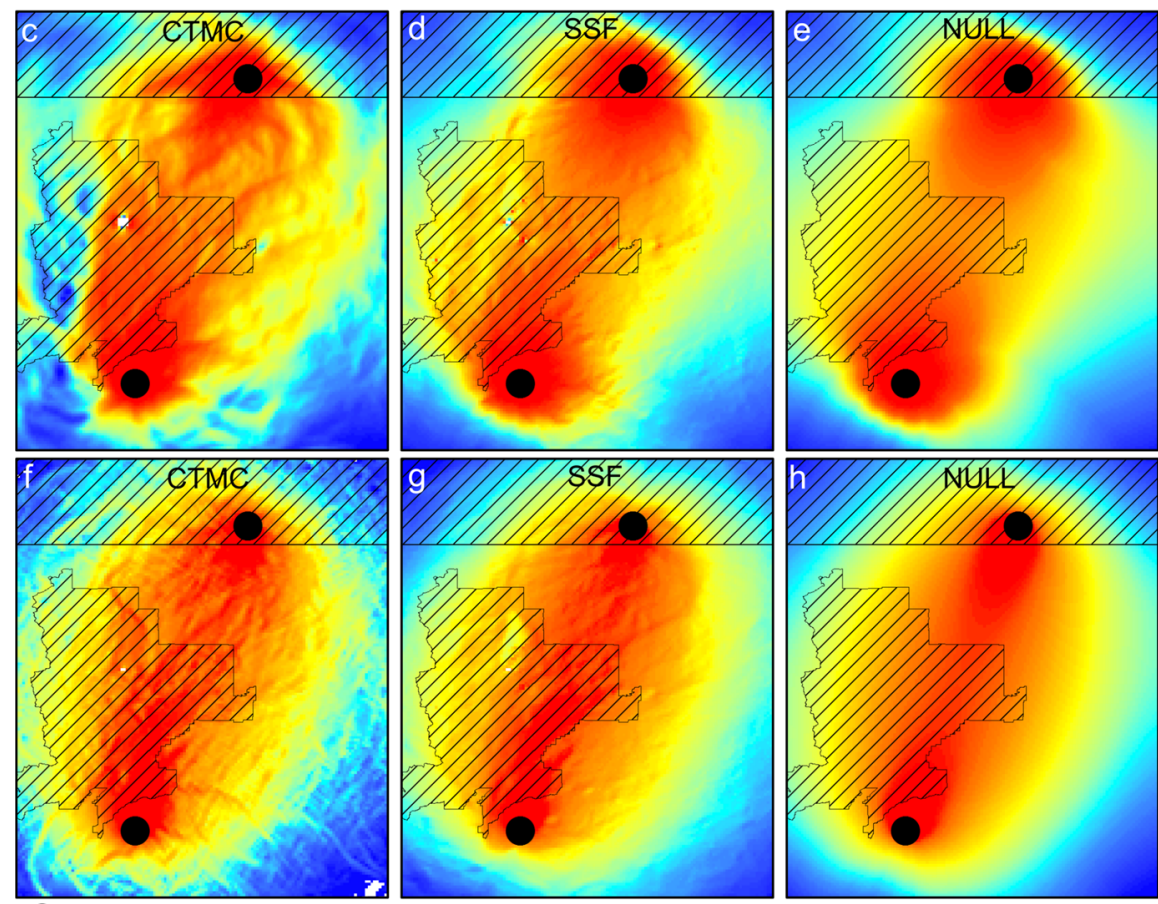

Seasonal Range(SR) Centroids

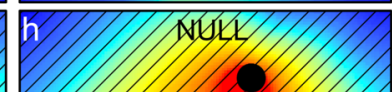

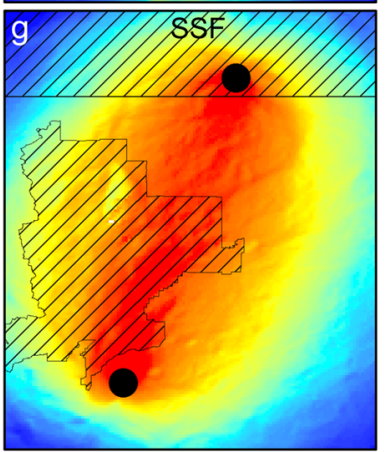

Connectivity Model Percentile

HIgh: 99 (between SR Centroids)

Low: 1 (along panels' perimeter) corridor detail than null models (Fig. 2). SSFs are frequently applied in studies of movement and resource use (e.g., Fortin et al. 2005, Thurfjell et al. 2014) and have been used to estimate resistance and inform connectivity models with relative success (Squires et al. 2013; Abrahms et al. 2016; Panzacchi et al. 2016; Zeller et al. 2016). The high level of accuracy from SSF-informed connectivity models in our study may be due to frequent bouts of stopping and foraging during migration. Stopover locations, where animals move slower and spend time foraging, have been recognized as essential stepping stones in the migration process (Sawyer and Kauffman 2011; Aikens et al. 2017), and can result in increased fat stores during the growing season (Middleton et al. 2018). Because such stopovers may result in valuable predictions from SSFs, it is important that researchers and practitioners consider both routine-slow and rarefast movements to understand the relative importance of those behaviors to overall connectivity (Van Dyck and Baguette 2005). It will also be important to test CTMC and SSF approaches on other species and other types of movement (e.g., dispersal) and use of corridors (e.g., movement versus habitat) to fully 

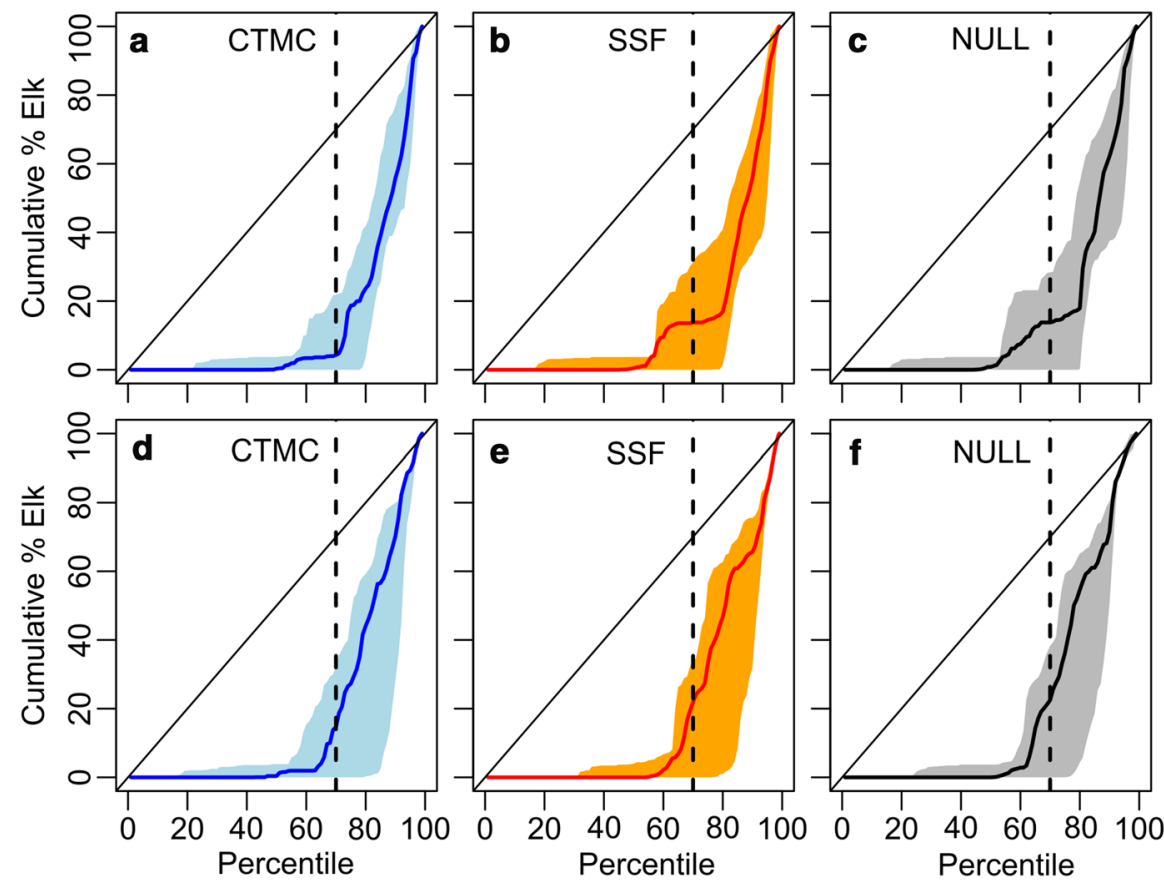

Fig. 3 Comparison of the cumulative percent of validation elk GPS locations found within each percentile of $\mathbf{a}-\mathbf{c}$ circuit theory and d-f shortest random path algorithms applied to the continuous time Markov chain (CTMC), step selection function

(SSF), and null (NULL) resistances. Solid lines beneath the diagonal depict the median of four validation elk and shaded regions depict the full range for those elk. Dotted vertical lines indicate the 70 th percentile
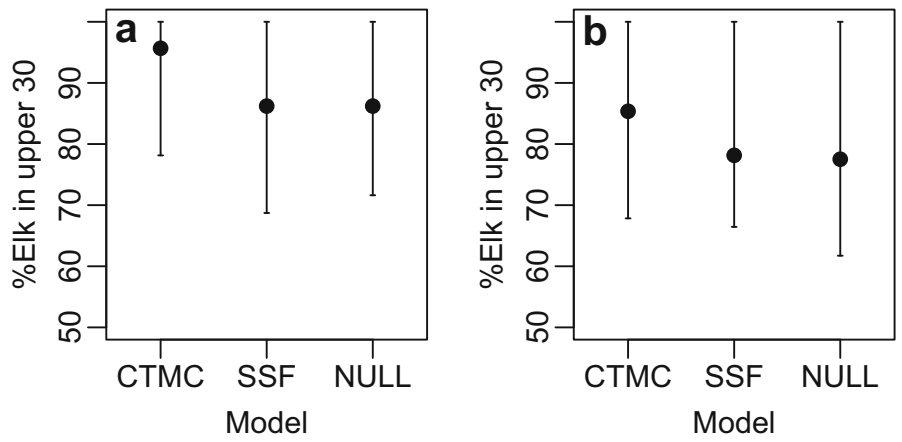

Fig. 4 Comparison of the cumulative percent of elk points found in the upper 30th percentiles (referred to as upper 30) of $\mathbf{a}$ circuit theory and $\mathbf{b}$ shortest random path algorithms applied to the continuous time Markov chain (CTMC), step selection

appreciate the differences in speed versus selection in studies of movement and connectivity.

The SSF habitat suitability surface in our analysis did not account for local changes in resource availability, and thus represented population-level habitat suitability assuming all terrain was available. We did not expect this to affect the comparison of CTMC and SSFs because these two approaches model different

function (SSF), and null (NULL) resistances. Dots represent the median of four validation elk and whiskers represent the full range for those elk

aspects of movement (e.g., speed vs. selection, respectively), and areas that facilitate fast movement were expected to be important for predicting elk migration corridors. Several studies, however, have mapped SSF habitat suitability in a way that accounts for local changes in resource availability. One method uses differences in the proportion of land cover types between each pixel and an availability kernel centered 
on that pixel to adjust gridded habitat suitability values based on the local context of the landscape (Zeller et al. 2016). However, this method may necessitate multiple models with different kernel sizes to effectively understand the scale that animals are responding to the landscape (Zeller et al. 2014). Other methods have involved simulating elk movement using the past track of each animal and changes in local availability (Signer et al. 2017) or using a master equation of space use (Merkle et al. 2017a, b), but these are highly computationally intensive (Avgar et al. 2016; Signer et al. 2017) and to our knowledge they have not been used to estimate landscape resistance.

In addition to testing differences in speed and selection, we examined two connectivity modeling algorithms that model movement differently. Previous work suggests that elk move with some knowledge of their landscape (Wolf et al. 2009; McClure et al. 2016), and therefore we expected shortest random path (SRP) models used to constrain random movement between grid cells to predict elk locations more accurately than circuit theory algorithms based on random walk theory. Contrary to this expectation, our analysis found that circuit theory predicted elk locations better than SRP models, though there was substantial overlap in the range of prediction accuracy (Figs. 3, 4) suggesting that elk migration corridors are explained by some degree of random walk (i.e., explorative) and optimized movement (i.e., minimizes cost of traveling). As previous work found that explorative movements can be necessary to maximize foraging efficiency in bison (Merkle et al. 2017b), a mix of movement types is not unexpected for migrating elk that also need to maximize efficiency while foraging at various stopovers during the migration.

Although the accuracy of predictions from connectivity models was high, it is important to note that elk in this study followed a relatively direct route during at least the first half of the migration (Fig. 2). This pattern of direct migration over long distances (as a broad scale pattern; different from fine scale fast, directed movements between relocations) provides an explanation for null model success, and highlights the importance of knowledge about elk winter and summer range locations to studies of elk migration and connectivity. Corridor prediction accuracy could decline, however, for elk with less direct migratory routes even for models informed by landscape resistance. SSF resistance, for example, did little to improve prediction accuracy of the less direct routes compared to null model predictions (Fig. 2), and CTMC resistance showed only moderate improvements overall. Estimating a more informative resistance surface may be difficult because elk can travel across most habitat types, and their movement is also likely to be affected by other factors not examined via landscape resistance (i.e., predation risk; Fortin et al. 2005). In addition, ungulates can exhibit strong memory capabilities (Merkle et al. 2014), and evidence suggests that their past experience influences future movements (Wolf et al. 2009). Indeed, elk exhibit fidelity to traditional migration routes and seasonal ranges, though with some plasticity over time (Van Dyke et al. 1998; Eggeman et al. 2016). Incorporating memory, however, is not trivial, in part because the memory process is hidden and current simulation methods extended to this process are highly computationally intensive (Fagan et al. 2013; Merkle et al. 2017b). While identifying source and target nodes is a step toward explicitly incorporating memory into connectivity models, it may still be challenging to accurately simulate elk movement along less direct migration routes. Future studies should examine CTMC and SSF-informed connectivity models across a range of species and migratory elk populations to understand the effect that less direct migration routes can have on prediction accuracy.

In conclusion, animals are likely to move faster through undesirable habitats and slower in preferred habitats. As a result, studies of movement rate and resource selection are likely to reach opposite conclusions regarding habitat preferences during movement. Prediction of migration and dispersal corridors where faster, more directed movements can occur is often achieved using resistance surfaces derived from studies of resource selection (e.g., Zeller et al. 2012; Abrahms et al. 2016), but the focus on selection rather than speed could affect our ability to accurately predict where animals move and result in conservative corridor predictions that exclude areas important for connectivity. We found CTMC-informed connectivity models to be a more realistic alternative to SSFinformed connectivity models for studying elk migration corridors and connectivity in western Wyoming, and thus recommend the addition of CTMC into the methodological toolkit of connectivity modeling and conservation. 
Acknowledgements We thank the U.S. Geological Survey for funding this project. We thank the Wyoming Game and Fish Department, National Elk Refuge and Grand Teton National Park for providing elk data. We also thank three anonymous reviewers for providing valuable comments. Any use of trade, firm, or product names is for descriptive purposes only and does not imply endorsement by the U.S. Government.

Open Access This article is distributed under the terms of the Creative Commons Attribution 4.0 International License (http:// creativecommons.org/licenses/by/4.0/), which permits unrestricted use, distribution, and reproduction in any medium, provided you give appropriate credit to the original author(s) and the source, provide a link to the Creative Commons license, and indicate if changes were made.

\section{References}

Abrahms B, Sawyer SC, Jordan NR, McNutt JW, Wilson AM, Brashares JS (2016) Does wildlife resource selection accurately inform corridor conservation? J Appl Ecol. https://doi.org/10.1111/1365-2664.12714

Aikens EO, Kauffman MJ, Merkle JA, Dwinnell SPH, Fralick GL, Monteith KL (2017) The greenscape shapes surfing of resource waves in a large migratory herbivore. Ecol Lett 20:741-750

Avgar T, Potts JR, Lewis MA, Boyce MS (2016) Integrated step selection analysis: bridging the gap between resource selection and animal movement. Methods Ecol Evol 7:619-630

Beier P, Majka DR, Spencer WD (2008) Forks in the road: choices in procedures for designing wildland linkages. Conserv Biol 22:836-851

Bennett AF (2003) Linkages in the landscape: the role of corridors and connectivity in wildlife conservation. IUCN, Gland/Cambridge

Blazquez-Cabrera S, Gastón A, Beier P, Garrote G, Simón MA, Saura S (2016) Influence of separating home range and dispersal movements on characterizing corridors and effective distances. Landscape Ecol 31:2355-2366

Brennan A, Courtemanch AB, Cole EK, Dewey SR, Cross PC (2018) Elk GPS collar data from national Elk refuge (2006-2015): U.S. Geological Survey data release. https:// doi.org/10.5066/F7FF3RNW

Chetkiewicz CLB, Boyce MS (2009) Use of resource selection functions to identify conservation corridors. J Appl Ecol 46:1036-1047

Chetkiewicz C-LB, St. Clair CC, Boyce MS (2006) Corridors for conservation: integrating pattern and process. Annu Rev Ecol Evol Syst 37:317-342

Cole EK, Foley AM, Warren JM, Smith BL, Dewey SR, Brimeyer DG, Fairbanks WS, Sawyer H, Cross PC (2015) Changing migratory patterns in the Jackson Elk Herd. J Wildl Manage 79:877-886

Eggeman SL, Hebblewhite M, Bohm H, Whittington J, Merrill EH (2016) Behavioural flexibility in migratory behaviour in a long-lived large herbivore. J Anim Ecol 85:785-797
Elliot NB, Cushman SA, Macdonald DW, Loveridge AJ (2014) The devil is in the dispersers: predictions of landscape connectivity change with demography. J Appl Ecol 51:1169-1178

Fagan WF, Lewis MA, Auger-Méthé M, Avgar T, Benhamou S, Breed G, LaDage L, Schlägel UE, Tang W, Papastamatiou YP, Forester J, Mueller T (2013) Spatial memory and animal movement. Ecol Lett 16:1316-1329

Forester JD, Im HK, Rathouz PJ (2009) Accounting for animal movement in estimation of resource selection functions: sampling and data analysis. Ecology 90:3554-3565

Fortin D, Beyer HL, Boyce MS, Smith DW (2005) Wolves influence elk movements: behavior shapes a trophic cascade in Yellowstone National Park. Ecology 86:1320-1330

Hanks EM (2017) ctmcmove: modeling animal movement with continuous-time discrete-space Markov chains. R package version 1.2.8. https://CRAN.R-project.org/package= ctmomove

Hanks EM, Hooten MB (2013) Circuit theory and model-based inference for landscape connectivity. J Am Stat Assoc 108:22-33

Hanks EM, Hooten MB, Alldredge MW (2015) Continuoustime discrete-space models for animal movement. Ann Appl Stat 9:145-165

Hanks EM, Hooten MB, Johnson DS, Sterling JT (2011) Velocity-based movement modeling for individual and population level inference. PLoS ONE. https://doi.org/10. 1371/journal.pone.0022795

Heller NE, Zavaleta ES (2009) Biodiversity management in the face of climate change: a review of 22 years of recommendations. Biol Conserv 142:14-32

Hilty JA, Lidicker WZJ, Merenlender A (2006) Corridor ecology: the science and practice of linking landscapes for biodiversity conservation. Island Press, Washington, DC

Houston DB (1982) The Northern Yellowstone elk: ecology and management. Macmillan, New York

Johnson CJ, Parker KL, Heard DC, Gillingham MP (2002) Movement parameters of ungulates and scale-specific responses to the environment. J Anim Ecol 71:225-235

Keeley ATH, Beier P, Gagnon JW (2016) Estimating landscape resistance from habitat suitability: effects of data source and nonlinearities. Landscape Ecol 31:2151-2162

Keeley ATH, Beier P, Keeley BW, Fagan ME (2017) Habitat suitability is a poor proxy for landscape connectivity during dispersal and mating movements. Landsc Urban Plan 161:90-102

Kivimäki I, Shimbo M, Saerens M (2014) Developments in the theory of randomized shortest paths with a comparison of graph node distances. Phys A Stat Mech Appl 393:600-616

Manly BFJ, McDonald LL, Thomas DL, McDonald TL, Erickson WP (2002) Resource selection by animals: statistical design and analysis for field studies, 2nd edn. Kluwer, Amsterdam

McClure ML, Hansen AJ, Inman RM (2016) Connecting models to movements: testing connectivity model predictions against empirical migration and dispersal data. Landscape Ecol 31:1419-1432

McRae B, Dickson B, Keitt T, Shah V (2008) Using circuit theory to model connectivity in ecology, evolution, and conservation. Ecology 89:2712-2724 
McRae BH, Shah V, Mohapatra T (2013) Circuitscape 4 user guide. The Nature Conservancy. http://www.circuitscape. org

Merkle JA, Cross PC, Scurlock BM, Cole EK, Courtemanch AB, Dewey SR, Kauffman MJ (2017a) Linking spring phenology with mechanistic models of host movement to predict disease transmission risk. J Appl Ecol. https://doi. org/10.1111/1365-2664.13022

Merkle JA, Fortin D, Morales JM (2014) A memory-based foraging tactic reveals an adaptive mechanism for restricted space use. Ecol Lett 17:924-931

Merkle JA, Potts JR, Fortin D (2017b) Energy benefits and emergent space use patterns of an empirically parameterized model of memory-based patch selection. Oikos. https://doi.org/10.1111/oik.03356

Middleton AD, Kauffman MJ, McWhirter DE, Cook JG, Cook RC, Nelson AA, Jimenez MD, Klaver RW (2013) Animal migration amid shifting patterns of phenology and predation: lessons from a Yellowstone elk herd. Ecol 94:1245-1256

Middleton AD, Merkle JA, McWhirter DE, Cook JG, Cook RC, White PJ, Kauffman MJ (2018) Green-wave surfing increases fat gain in a migratory ungulate. Oikos. https:// doi.org/10.1111/oik.05227

Morris LR, Proffitt KM, Blackburn JK (2016) Mapping resource selection functions in wildlife studies: concerns and recommendations. Appl Geogr 76:173-183

Panzacchi M, Van Moorter B, Strand O, Saerens M, Kivimäki I, St. Clair CC, Herfindal I, Boitani L (2016) Predicting the continuum between corridors and barriers to animal movements using step selection functions and randomized shortest paths. J Anim Ecol 85:32-42

Pettorelli N, Vik JO, Mysterud A, Gaillard J, Tucker CJ, Stenseth NC (2005) Using the satellite-derived NDVI to assess ecological responses to environmental change. Trends Ecol Evol 20:503-510

R Core Development Team (2017) R: a language and environment for statistical computing. R Foundation for Statistical Computing, Vienna

Saerens M, Achbany Y, Fouss F, Yen L (2009) Randomized shortest-path problems: two related models. Neural Comput 21:2363-2404

Sawyer H, Kauffman MJ (2011) Stopover ecology of a migratory ungulate. J Anim Ecol 80:1078-1087
Scharf H, Hooten MB, Johnson DS (2017) Imputation approaches for animal movement modeling. J Agric Biol Environ Stat 22:335-352

Signer J, Fieberg J, Avgar T (2017) Estimating utilization distributions from fitted step-selection functions. Ecosphere. https://doi.org/10.1002/ecs2.1771

Squires JR, DeCesare NJ, Olson LE, Kolbe JA, Hebblewhite M, Parks SA (2013) Combining resource selection and movement behavior to predict corridors for Canada lynx at their southern range periphery. Biol Conserv 157:187-195

Therneau T (2017) Survival: survival analysis. R package version 2.38. https://CRAN.R-project.org/package=survival

Thurfjell H, Ciuti S, Boyce MS (2014) Applications of stepselection functions in ecology and conservation. Mov Ecol $2: 4$

Trainor AM, Walters JR, Morris WF, Sexton J, Moody A (2013) Empirical estimation of dispersal resistance surfaces: a case study with red-cockaded woodpeckers. Landscape Ecol 28:755-767

Van Dyck H, Baguette M (2005) Dispersal behaviour in fragmented landscapes: routine or special movements? Basic Appl Ecol 6:535-545

Van Dyke FG, Klein WC, Stewart ST (1998) Long-term range fidelity in Rocky Mountain Elk. J Wildl Manage 62:1020-1035

van Etten J (2017) R Package gdistance: distances and routes on geographical grids. J Stat Softw. https://doi.org/10.18637/ jss.v076.i13

Wolf M, Frair J, Merrill E, Turchin P (2009) The attraction of the known: the importance of spatial familiarity in habitat selection in wapiti Cervus elaphus. Ecography (Cop) 32:401-410

Zeller KA, McGarigal K, Beier P, Cushman SA, Vickers TW, Boyce WM (2014) Sensitivity of landscape resistance estimates based on point selection functions to scale and behavioral state: pumas as a case study. Landscape Ecol 29:541-557

Zeller KA, McGarigal K, Cushman SA, Beier P, Vickers TW, Boyce WM (2016) Using step and path selection functions for estimating resistance to movement: pumas as a case study. Landscape Ecol 31:1319-1335

Zeller KA, McGarigal K, Whiteley AR (2012) Estimating landscape resistance to movement: a review. Landscape Ecol 27:777-797 